Research Article

\title{
An Improved CEEMDAN Time-Domain Energy Entropy Method for the Failure Mode Identification of the Rolling Bearing
}

\author{
Fengfeng Bie $\mathbb{D}^{\text {, }}$, Yi Miao $(\mathbb{D}$, Fengxia Lyu $\mathbb{i}$, Jian Peng $\mathbb{D}$, and Yue Guo \\ School of Mechanical Engineering, Changzhou University, Changzhou 213016, Jiangsu, China \\ Correspondence should be addressed to Fengfeng Bie; bieff@cczu.edu.cn
}

Received 25 August 2021; Revised 28 September 2021; Accepted 26 October 2021; Published 9 November 2021

Academic Editor: Wahyu Caesarendra

Copyright (C) 2021 Fengfeng Bie et al. This is an open access article distributed under the Creative Commons Attribution License, which permits unrestricted use, distribution, and reproduction in any medium, provided the original work is properly cited.

\begin{abstract}
As a key component of a mechanical system, the extraction and accurate identification of rolling bearing fault feature information are of great importance to guarantee the normal operation of the mechanical system. Aiming at that the extraction of rolling bearing fault features and traditional support vector machine parameters affects the overall accuracy of pattern classification, the improved CEEMDAN (complete ensemble empirical mode decomposition with adaptive noise) time-domain energy entropybased model for fault pattern recognition is proposed. The ICEEMDAN method is developed to decompose the signal to obtain the IMF component series. Then, the particular IMF components are selected according to the trend of correlation coefficient and variance contribution rate; meanwhile, the information entropy (power spectral entropy, singular spectral entropy, and timedomain energy entropy) of the screened IMF components is calculated to construct the feature vector sets, respectively. Finally, the feature vector sets are input into the PSO-SVM (particle swarm optimization-support vector machine) based model for training and pattern recognition. The effectiveness of the proposed method of the improved CEEMDAN time-domain energy entropy and PSO-SVM model is testified through numerical simulation and experiments on rolling bearing datasets. The comparison proceeded with the other mainstream intelligent recognition techniques indicates the superiority of the method with the diagnostic accuracy of $100 \%$ as for the final validation.
\end{abstract}

\section{Introduction}

As a critical part of rotating machinery, the condition of rolling bearings directly could affect the operation of the entire system [1]. Therefore, accurate judgment of the health status of rolling bearings is essential to improve the reliability and ensure the safe operation of the equipment $[2,3]$. Extracting fault features from the original collected vibration signals and pattern recognition are critical for the fault diagnosis of rolling bearings. Although the traditional frequency domain analysis method can point out certain characteristics of rolling bearing faults, it is limited in determining the degree of damage in the operation process. In recent years, some methods based on adaptive signal decomposition have subsequently attracted the attention of researchers. Several calculation models have been developed and implemented. The empirical mode decomposition (EMD) algorithm can decompose the signal adaptively into intrinsic mode functions (IMFs) of different scales as well as residual components [4], which can be analyzed independently for different components [5]. Applying EMD and entire empirical mode decomposition (EEMD) to bearing data can identify the external raceway failure of the slewing ring and the failure frequency of rolling elements [6]. However, the algorithm is constrained with a strict mathematical foundation with low computational efficiency; moreover, it is prone to render modal mixing with spurious components. On this point, $\mathrm{Wu}$ and Huang [7] proposed the EEMD, which could suppress the modal mixing phenomenon to some extent; however, the algorithm is time-consuming, leading to low efficiency in the process. Torres et al. proposed the adaptive complete ensemble empirical mode decomposition (CEEMDAN) method $[8,9]$, which could effectively reduce the reconstruction error and improve the decomposition efficiency; nevertheless, it could not completely solve the problem of modal mixing and the spurious 
components generated in the signal decomposition process. Based on this point, an improved self-adaptive complete ensemble empirical mode decomposition (ICEEMDAN) algorithm is developed to complete the feature extraction from the original vibration signal.

For the pattern recognition section, it is necessary to design a multimode classifier for the extracted features by the ICEEMDAN method on the rolling bearing vibration signal. In terms of mechanical equipment failure pattern recognition, the support vector machine (SVM) classification technology and artificial neural network (ANN) [10] are commonly used. However, the support vector machine is applicable for local extreme value, small sample, nonlinearity, or high dimensionality failure modes which might be encountered in the machine learning classification [11]. In the SVM fault classification process, determining the appropriate kernel function, penalty factor $c$, and kernel function parameter $g$ plays a vital role in the accuracy of subsequent diagnostic results. In order to promote the performance of the diagnostic model, optimization algorithms are employed to optimize the parameters to obtain the optimal parameter values. Wen et al. used an improved grid search algorithm to optimize the number of decision trees and the number of candidate split attributes in the random forest algorithm [12]. Liu and others proposed the GA-SVM model to locate and detect bridge ripples [13]. The support vector machine (SVM) model classifier based on particle swarm optimization (PSO) is built for rolling bearing fault classification on account of the advantages of the PSO algorithm, including faster search speed, high efficiency, and strong convergence ability $[14,15]$. In the model application, the set of feature vectors constructed from different states of rolling bearings is input into the PSO-SVM model to complete the pattern recognition.

The rest of this article is organized in the following manner: in Section 2, the theoretical foundation of the principles of the improved adaptive complete ensemble empirical mode decomposition (ICEEMDAN) algorithm, the screening criterion of the correlation coefficient-variance contribution rate, the information entropy, and the method based on the PSO-SVM model are reviewed and discussed. Section 3 is the numerical simulation section, in which the model is established in detail, the simulated signals are achieved, and the application of the proposed method to derive the pattern recognition results is implemented. Section 4 is the experimental verification part, where the validity of the proposed method is verified by experimentally measuring the signals and processing them. Section 5 draws conclusions and recommendations.

\section{Principle and Method}

2.1. Algorithm of ICEEMDAN. The adaptive complete ensemble empirical mode decomposition (CEEMDAN) is a major improvement algorithm of EEMD [16], where the positive and negative pairs of complementary white noise are added, thereby effectively suppressing the noise problem in the reconstruction process and improving the decomposition efficiency [17]. However, a small amount of residual noise and false components were produced in the decomposition process of the ICEEMDAN. To address this issue, the improved CEEMDAN (ICEEMDAN) algorithm was proposed by Colominas et al. [18], which could greatly suppress the residual noise and spurious components in the decomposition process. The specific decomposition process is as follows:

(1) Gaussian white noise is added to the original signal.

$$
\mathbf{X}_{1}^{(i)}=x+\mathbf{e}_{1} \mathbf{E}_{1}\left(\boldsymbol{\omega}^{(i)}\right), \quad I=(1,2 \ldots N)
$$

where $x$ is the original signal, $\mathbf{e}_{1}$ is the expected signal-to-noise ratio of the first decomposed signal, $\omega^{(i)}$ is the $i$ th added Gaussian white noise, and $\mathbf{E}_{1}(\cdot)$ denotes the first IMF after EMD decomposition.

(2) Calculate the first decomposition residuals.

$$
r_{1}=\left\langle\mathbf{X}_{1}^{(i)}-\mathbf{E}_{1}\left(\mathbf{X}_{1}^{(i)}\right)\right\rangle
$$

where $\langle\cdot\rangle$ denotes the averaging sign.

(3) Calculate the first modal component $\mathbf{I M F}_{1}$.

$$
\mathbf{I M F}_{1}=x-r_{1} \text {. }
$$

(4) Estimate the second residual as a series of mean values of $r_{1}+e_{2} E_{2}\left(\omega^{(i)}\right)$, and define the second modal component $\mathbf{I M F}_{2}$ :

$$
\mathbf{I M F}_{2}=r_{1}-r_{2}=r_{1}-\left\langle r_{1}+e_{2} E_{2}\left(\omega^{(i)}\right)\right\rangle
$$

where $\mathbf{e}_{2}$ is the expected signal-to-noise ratio of the second decomposed signal.

(5) Calculate the $k$ th-order residual $\mathbf{r}_{k}$.

$$
\mathbf{r}_{k}=\mathbf{X}_{k}^{(i)}-\mathbf{E}_{k}\left(\mathbf{X}_{k}^{(i)}\right) \text {. }
$$

(6) Calculate the $k$ th modal component $\mathbf{I M F}_{k}$.

$$
\mathbf{I M F}_{k}=\mathbf{r}_{k}-\mathbf{r}_{k} \text {. }
$$

(7) Return to Step 5 to calculate $\mathbf{r}_{k+1}$.

2.2. Correlation Coefficient-Variance Contribution of Screening Guidelines. Not all the IMF components generated after the original signal are decomposed by ICEEMDAN which can characterize the original signal. Hence, in order to accurately identify the fault categories, the spurious components need to be removed. In this study, the correlation coefficient-variance contribution rate is employed as the screening criterion.

2.2.1. Correlation Coefficient. The correlation coefficient is a statistical quantity that is employed to characterize the degree of correlation between the variables [19], i.e., a larger correlation coefficient indicates a stronger correlation. The correlation analysis is executed between the original signal and each IMF component obtained after ICEEMDAN decomposition, and the larger calculated result indicates that 
the IMF component contains more feature information of the original signal.

The self-correlation coefficients of the original signal with each IMF component are calculated as follows:

$$
\mathbf{R}_{x}(m)=\frac{1}{N} \sum_{i=0}^{N-1} x(i) x(i+m) .
$$

The self-correlation function is normalized, and the correlation coefficient between the self-correlation function $\mathbf{R}_{\mathrm{IMF} 1}{ }^{(m)}, \mathbf{R}_{\mathrm{IMF} 1}^{(m)}, \ldots \ldots, \mathbf{R}_{\mathrm{IMF} 1}^{(m)}$ of each IMF component and the autocorrelation function $\mathbf{R}_{x}(m)$ of the original signal is calculated. The correlation coefficient is defined as

$$
\rho(j)=\frac{\sum_{i=1}^{2 N-1} \mathbf{R}_{I M F j}{ }^{(i)} \mathbf{R}_{x}(i)}{\sqrt{\sum_{i=1}^{2 N-1} \mathbf{R}^{2}{ }_{I M F j}^{(i)} \mathbf{R}_{x}^{2}(i)}},
$$

where $N$ is the number of signal sampling points and $j$ represents the $j$ th IMF component.

2.2.2. Variance Contribution Rate. The variance contribution rate is a statistic employed to characterize the relative importance of factors. The higher variance contribution of a factor indicates the greater interaction between that factor and the original signal. The signal is decomposed into $\mathbf{I M F}_{1}, \mathbf{I M F}_{1} \ldots \ldots \mathbf{I M F}_{n}$ by ICEEMDAN. The variance contribution rate of the $i$ th IMF component and the original signal is calculated as follows [20]:

$$
\operatorname{mseb}(i)=\frac{1 / N \sum_{j=0}^{N} \operatorname{IMF}_{i}^{2}(j)-\left[1 / N \sum_{j=0}^{N} \operatorname{IMF}_{i}(j)\right]^{2}}{\sum_{i=1}^{n}\left\{1 / N \sum_{j=0}^{N} \operatorname{IMF}_{i}^{2}(j)-\left[1 / N \sum_{j=0}^{N} \operatorname{IMF}_{i}(j)\right]^{2}\right\}},
$$

where $N$ denotes the length of the signal and $n$ denotes the number of IMF components.

2.3. Information Entropy. Information entropy actually reflects the chaotic degree of uncertain factors in a system [21]. It describes the complexity of the information contained in a vibration signal [22], i.e., more information entropy indicates a more complex original signal and greater uncertainty and randomness involved.

2.3.1. Time-Domain Energy Entropy. As an information entropy, the time-domain energy entropy reflects the complexity and uncertainty of the signal energy distributed in the time domain [23]. It can be specifically expressed as

$$
\mathbf{E}_{\text {tdee }}=-\sum_{n=1}^{N} \frac{\mathbf{E}_{n}}{\mathbf{E}_{\text {sum }}} \ln \frac{\mathbf{E}_{n}}{\mathbf{E}_{\text {sum }}}
$$

where $\mathbf{E}_{n}=\mathbf{x}_{n}^{2}$ is the energy of each data point of the signal, $\mathbf{E}_{\text {sum }}=\sum_{n=1}^{N} \mathbf{x}_{n}^{2}$ is the total energy, and $N$ is the number of signal sampling points.
2.3.2. Singular Spectral Entropy. The singular spectral entropy reflects the degree of uncertainty of each mode of the time-domain signal under singular spectral division, while valuable information from finite time series could be further obtained. The signal is decomposed by ICEEMDAN to obtain the qth-order IMF component and the residual component. The singular value decomposition of the obtained IMF components of the $i$ th order is denoted as $\mu_{i}$, and then the singular spectral entropy of the signal is denoted as

$$
\mathbf{E}_{s s e}=-\sum_{i=1}^{M} q_{i} \log _{q_{i}}
$$

where $q_{i}=\mu_{i} / \sum_{i=1}^{M} \mu_{i}$ denotes the weight of the singular value of the $i$ th IMF component in the whole spectrum of singular values.

2.3.3. Power Spectrum Entropy. The power spectrum reflects the variation of the signal in the frequency domain energy, and it allows to find the frequency components of different signals with limited data. Therefore, the power spectrum entropy can be used to characterize the energy distribution of the signal in the frequency domain. Assuming that $\mathbf{F}_{i}(\omega)$ is obtained by the DFT for each IMF, the power spectrum can be expressed as

$$
S(\omega)=\frac{1}{N}\left|\mathbf{F}_{i}(\omega)\right|^{2} \quad(\omega=1,2 \ldots N),
$$

where $S(1), S(2) \ldots \ldots S(N)$ is the fraction of the power energy in the frequency domain and the total power energy is $\mathbf{S}_{\text {sum }}=\sum_{\omega=1}^{N} S(\omega)$. The proportional distribution of the individual frequencies in the power spectrum is defined as the information probability distribution. The power spectrum entropy can be expressed as

$$
\mathbf{E}_{\mathrm{pse}}=-\sum_{\omega=1}^{N}\left(S(\omega) \mathbf{S}_{\mathrm{sum}}\right) \ln \frac{S(\omega)}{\mathbf{S}_{\mathrm{sum}}} .
$$

2.4. SVM Parameter Optimization Based on PSO. SVM algorithm could be considered as a machine learning classification method based on the theory of structural risk minimization. Determining the kernel parameter $g$ and the penalty factor $c$ in the kernel function is a key step in the SVM, and usually, these two parameters are determined by empirical or grid search methods, but neither of these methods could ensure the global optimal solution [24]. In contrast, the particle swarm algorithm can primarily initialize a set of random particles and then find the optimal solution by certain iterations [25]. The particle adjusts its speed and position during the iterative process based on its own and its companion's dispersion experience. Hence, the PSO-SVM model is designed to optimize the kernel parameter $g$ and penalty factor $c$ in the kernel function in the SVM by PSO, and the results obtained from the training set of the SVM are utilized as the adaptation to the test set for recognition. The specific steps are as follows: 


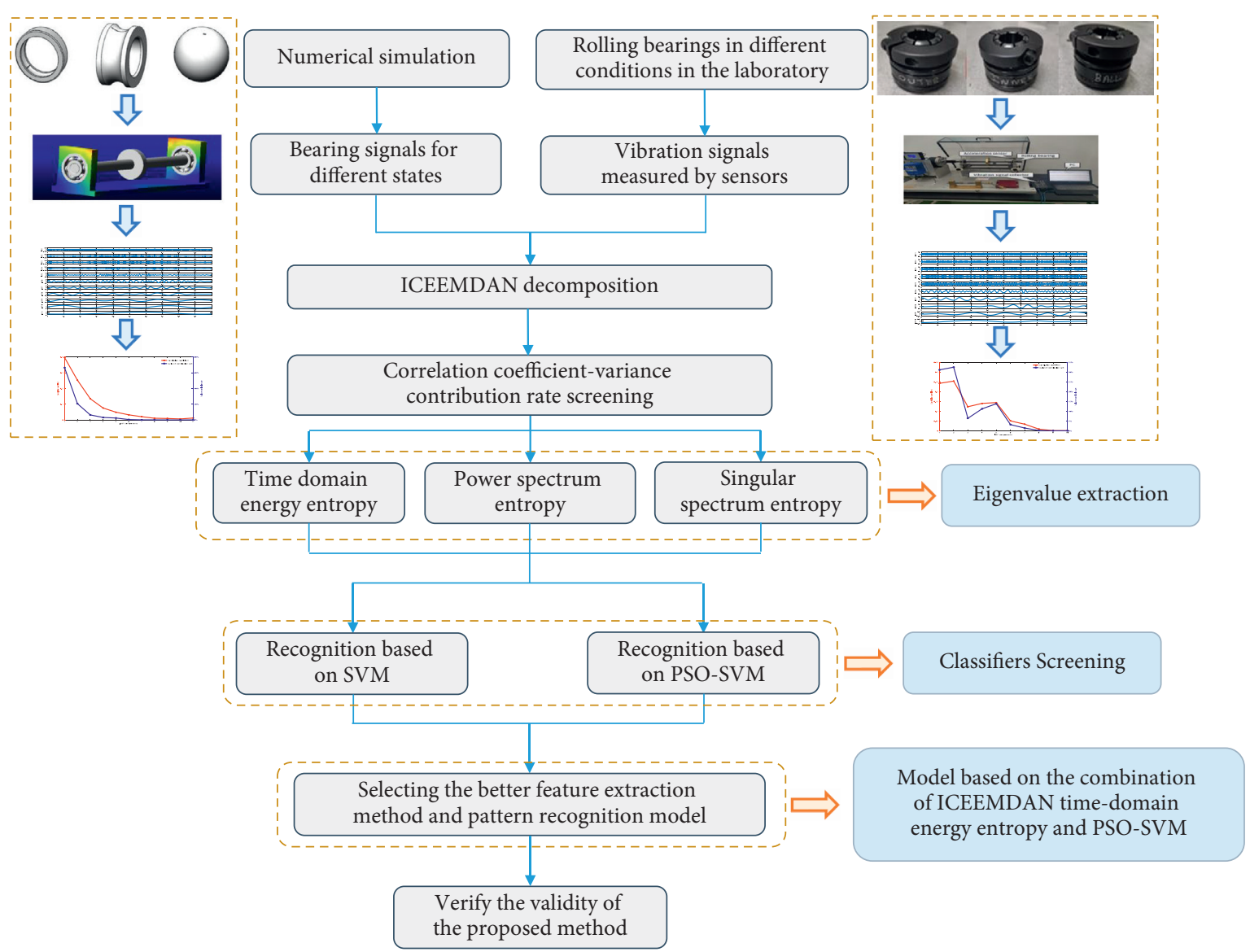

FiguRE 1: Research workflow.

(1) Initialization of a swarm of particles, which include two parameters of random position and velocity

(2) Training and testing in the SVM model to evaluate the fitness of each particle

(3) Finding the individual and global extremes of each particle and updating the velocity and position of the particle according to it

(4) Once the maximum number of iterations is reached, the optimal parameters $g$ and $c$ are obtained; if not, then go back to Step 2

2.5. Fault Diagnosis Process. In this study, the rolling bearing vibration signal obtained in numerical simulation is decomposed into a series of intrinsic mode functions (IMFs) with the ICEEMDAN decomposition algorithm. The IMF components that can characterize the original signal are selected by the correlation coefficient-covariance contribution criterion. Then, the information entropy (power spectral entropy, singular spectral entropy, and time-domain energy entropy) of these IMF components is calculated and constructed as feature vector sets, which are input into the SVM model and PSO-based SVM model for training and pattern recognition, respectively, for the comparison of feature extraction and pattern recognition. Finally, the signals of rolling bearings of different fault types in the laboratory were measured to complete the experimental
TABLE 1: Geometry dimensions of deep groove ball bearing 6406 .

\begin{tabular}{lc}
\hline Parameters & Numerical value \\
\hline Outer diameter of the outer ring $(\mathrm{mm})$ & 90 \\
Inner diameter of the outer ring $(\mathrm{mm})$ & 71.4 \\
Outer diameter of the inner ring $(\mathrm{mm})$ & 48.6 \\
Inner diameter of the inner ring $(\mathrm{mm})$ & 30 \\
Bearing mid-diameter $(\mathrm{mm})$ & 60 \\
Rolling element diameter $(\mathrm{mm})$ & 19.06 \\
Bearing width (mm) & 23 \\
Number of rolling elements/piece & 6 \\
Contact angle $\left(^{\circ}\right)$ & 0 \\
\hline
\end{tabular}

verification of the rolling bearing fault mode identification method based on the ICEEMDAN time-domain energy entropy model, and final conclusions were drawn. The research workflow of the proposed methodology is shown in Figure 1.

\section{Numerical Simulation}

In order to validate the effectiveness of the method proposed, the vibration of rolling bearings under different fault states is simulated through the ADAMS dynamics system. A typical 3D model of the rolling bearing simulation test bench was established using SolidWorks in the first place, and the model was imported into ADAMS for simulation subsequently. 


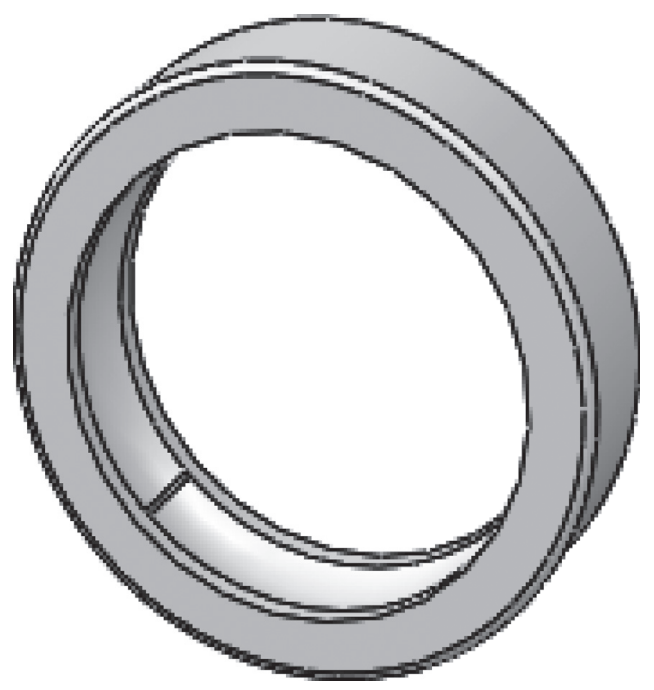

(a)

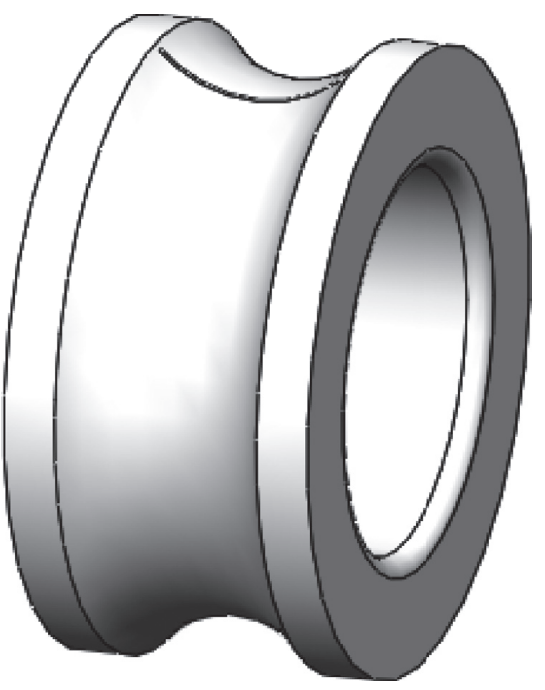

(b)

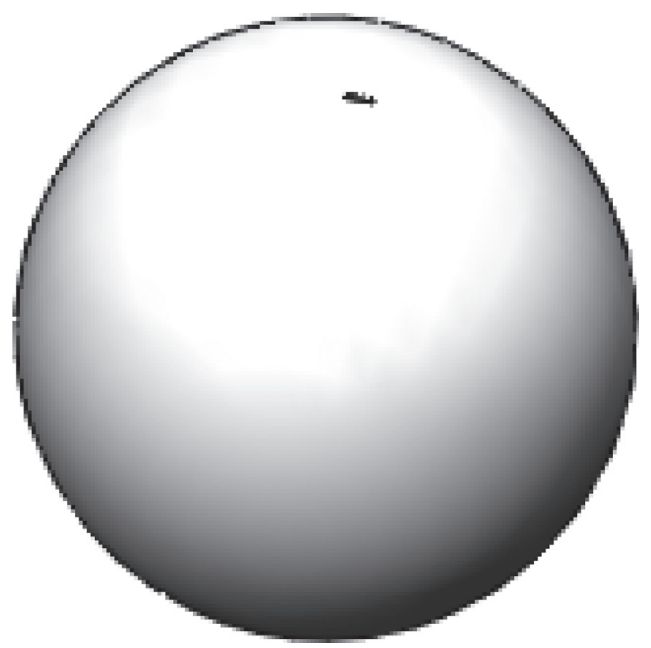

(c)

FIgURE 2: The fault model of rolling bearing parts. (a) Outer ring failure. (b) Inner ring failure. (c) Rolling element failure.

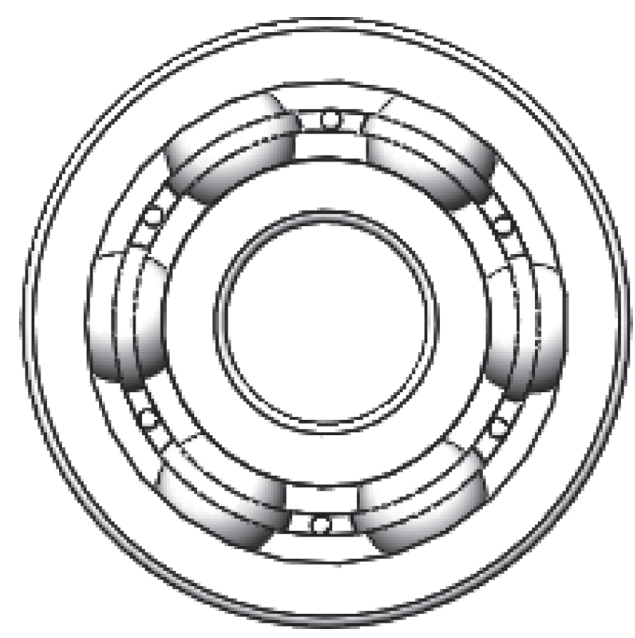

FIGURE 3: Rolling bearing model. 


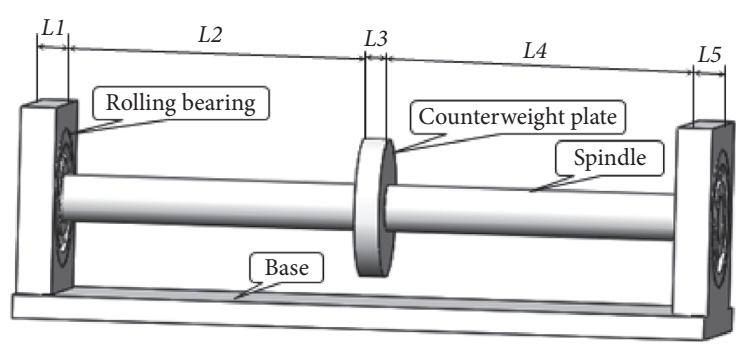

FIgURE 4: The rotor system model of the rolling bearing test bed.

TABLE 2: Simulation contact parameter setting.

\begin{tabular}{lccc}
\hline Parameters & $\begin{array}{c}\text { Between the rolling element and outer } \\
\text { ring }\end{array}$ & $\begin{array}{c}\text { Between the rolling element and inner } \\
\text { ring }\end{array}$ & $\begin{array}{c}\text { Between the rolling element and } \\
\text { cage }\end{array}$ \\
\hline Stiffness coefficient & $5.2 \mathrm{E} 5$ & $4.9 \mathrm{E} 5$ & $3.9 \mathrm{E} 5$ \\
Damping coefficient & $5.2 \mathrm{E} 3$ & $4.9 \mathrm{E} 3$ & $3.9 \mathrm{E} 3$ \\
Force index & 1.5 & 1.5 & 1.5 \\
Penetration depth & 0.1 & 0.1 & 0.1 \\
Dynamic friction & 0.05 & 0.05 & 0.05 \\
factor & 0.1 & 0.1 & 0.1 \\
Static friction factor & & 0 & \\
\hline
\end{tabular}

3.1. Rotor System-Bearing Dynamics Modeling. The deep groove ball bearing of type 6406 is set as the research object, whose structural parameter is shown in Table 1. The threedimensional model of the bearing and rotor system is established through SolidWorks, and a rectangular parallelepiped with a width of $1 \mathrm{~mm}$ and a depth of $0.5 \mathrm{~mm}$ is constructed on the basis of the normal outer ring, inner ring, and rolling elements of the rolling bearing to simulate the peeling failure of the rolling bearing as shown in Figure 2. The rolling bearing is composed of an outer ring, inner ring, rolling element, and cage, as shown in Figure 3. The rotor system of the test bench shown in Figure 4 consists of a base, a rotating shaft with a counterweight plate, and two rolling bearings, where $L 1=L 5=23 \mathrm{~mm} ; L 2=L 4=195 \mathrm{~mm}$; and $L 3=16 \mathrm{~mm}$.

\subsection{Dynamic Simulation of the Rolling Bearing Test Bench.} The three-dimensional modeling of the rolling bearing test stand was completed in SolidWorks, and the entire assembled model was imported into dynamics analysis software ADAMS for the simulation of the four modes after interference checking, i.e., normal condition, outer ring failure, inner ring failure, and rolling element failure. The model material is selected as steel; the fixed pairs are applied separately in the interfaces, including the bearing base and the ground, the bearing outer ring and the base, and the bearing inner ring and the shaft. The rolling element exerts contact forces with the outer ring, inner ring, and cage of the bearing [26, 27], with the specific parameters, as shown in Table 2 .

In order to ensure the simulation results close to the actual situation, the test bench base is optimized as flexible, i.e., the flexible body is replaced by the rigid body in ADAMS to convert it into a modal neutral file. Meanwhile, in order to simulate the operation of the rolling bearing realistically, a

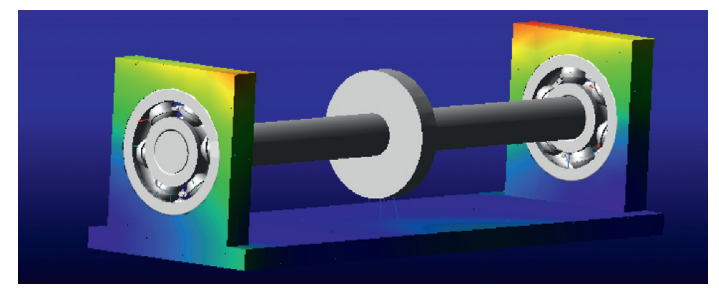

Figure 5: Dynamic simulation of the rolling bearing.

rotating sub is added to the rotating shaft, and the drive is supplied in the step as (time, $0,0 \mathrm{~d}$, and 1, 7200 d). Thus, the simulation is executed with the drive added, as shown in Figure 5. The simulation time is set as $1 \mathrm{~s}$, and the number of steps is 1000 steps.

After 30 sets of data were extracted, respectively, from the 4 typical modes, normal, outer ring failure, inner ring failure, and rolling body failure, totally 120 sets of data were achieved, among which $70 \%$ are randomly selected as training samples under different conditions, and 30\% are used as test samples. Then, the signal is decomposed by ICEEMDAN to obtain a series of IMF components, as shown in Figure 6. It could be observed from Figure 7 that the correlation coefficient-variance contribution rate of the first five-order IMF involves a positive trend. Therefore, the power spectral entropy, singular spectral entropy, and time-domain energy entropy of the first five orders of the IMFs are calculated separately as the set of feature vectors. The set of feature vectors of the respective entropy is input into the SVM model and PSO-SVM-based model for the final identification, respectively. In the training process, the normal state is set as label 1, the outer ring fault is label 2, the inner ring fault is label 3 , and the rolling element fault is label 4. As the power spectral entropy was selected as the feature, the recognition accuracy was $91.6 \%$ through the 

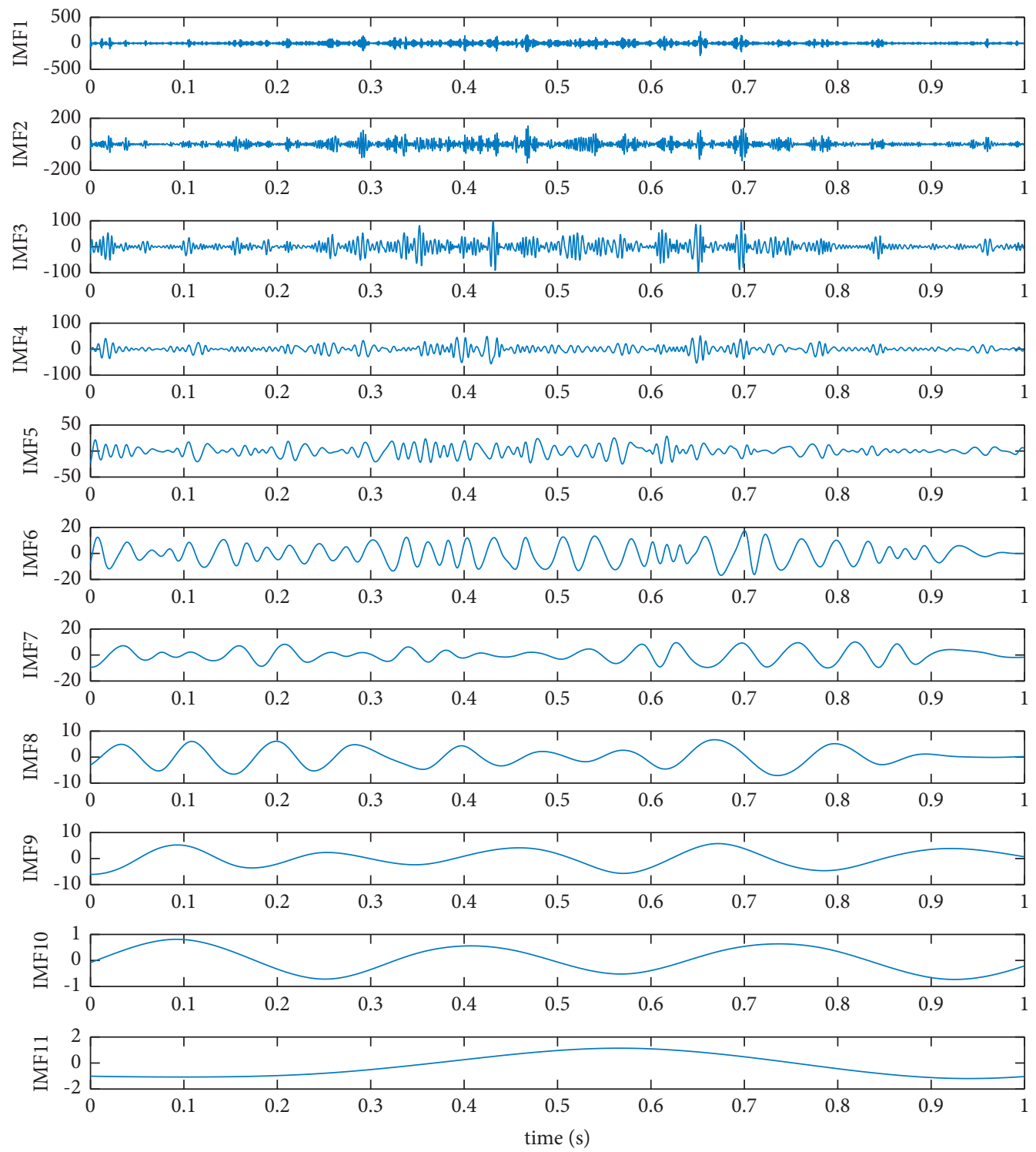

Figure 6: ICEEMDAN decomposition of the numerical simulation of outer ring failure.

SVM model and $88.9 \%$ through the PSO-SVM model for the 36 sets of data tested, as shown in Figure 8. As the singular spectrum entropy is selected for the feature vector as shown in Figure 9, 8 sets of data in the SVM model are not recognized, and the accuracy rate is merely $77.8 \%$, and the recognition accuracy rate based on the PSO-SVM model reaches $91.6 \%$, while as the time-domain energy entropy is selected as the feature as shown in Figure 10, the SVM model has 2 sets of data that are not recognized, with an accuracy rate of $94.4 \%$, and the recognition accuracy rate based on the PSO-SVM model reaches $100 \%$. It can be found that when singular spectral entropy and power spectral entropy are selected as features, the recognition effect is not superior to time-domain energy entropy in both cases.

\section{Experimental Validation}

The experiment is built on the SQI-MFS mechanical failure comprehensive simulation experiment platform. The vibration signals are collected by using the rolling bearing kits involving normal state, outer ring failure, inner ring failure, and rolling element failure in the laboratory. As shown in Figure 11, an acceleration sensor with a sensitivity of $102 \mathrm{mv} / \mathrm{g}$, IOtech $640 \mathrm{U}$ dynamic signal analyzer, and a laptop are employed in the experiment system. The sampling frequency of the vibration signal is set to $5120 \mathrm{~Hz}$, and the rotation frequency of the shaft is $20 \mathrm{~Hz}$.

In order to compare with the aforementioned numerical simulation results, 40 sets of data are extracted for normal in the experiment, outer ring failure, inner ring failure, and 


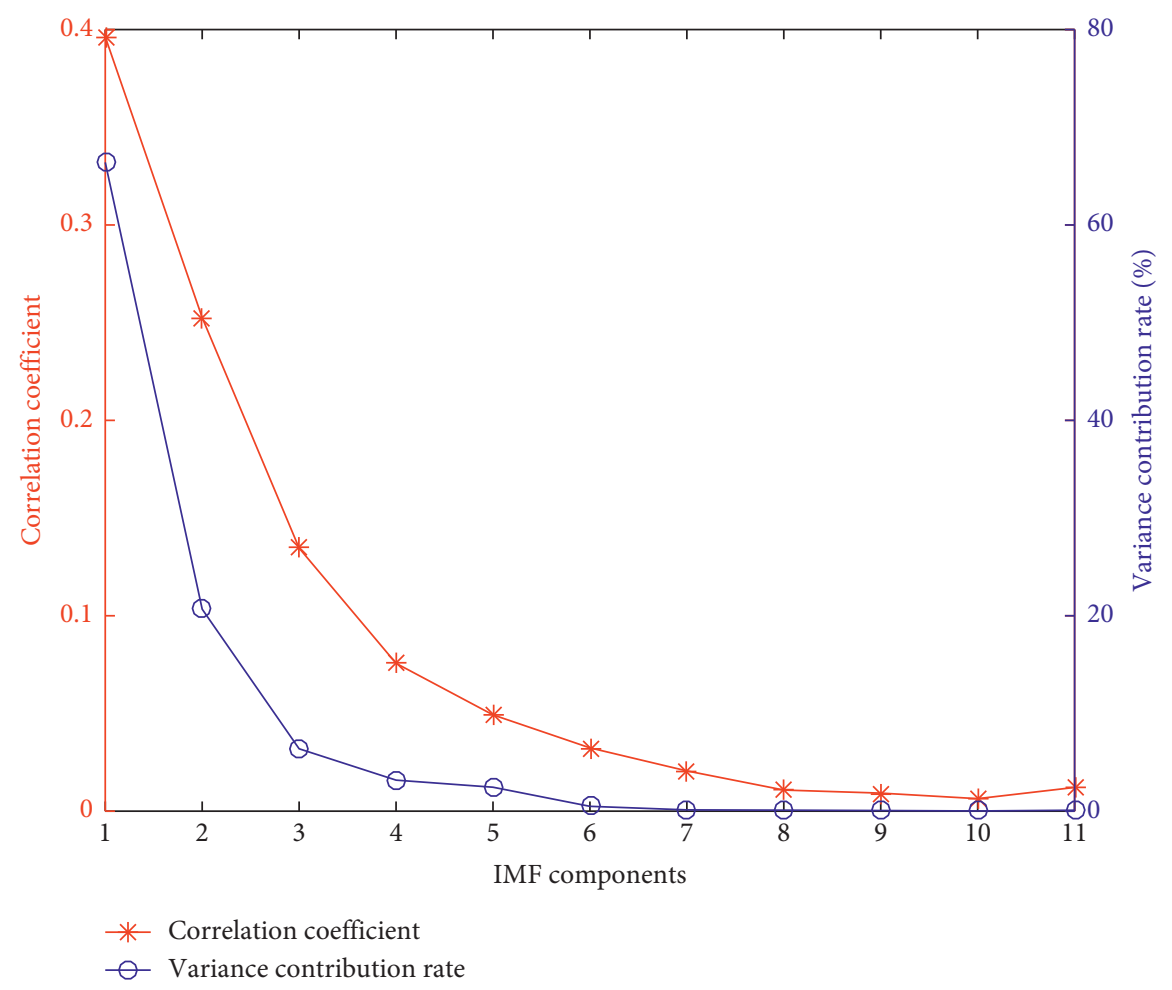

FIgURE 7: Correlation coefficient-variance contribution trend of the numerical simulation of the outer ring fault.

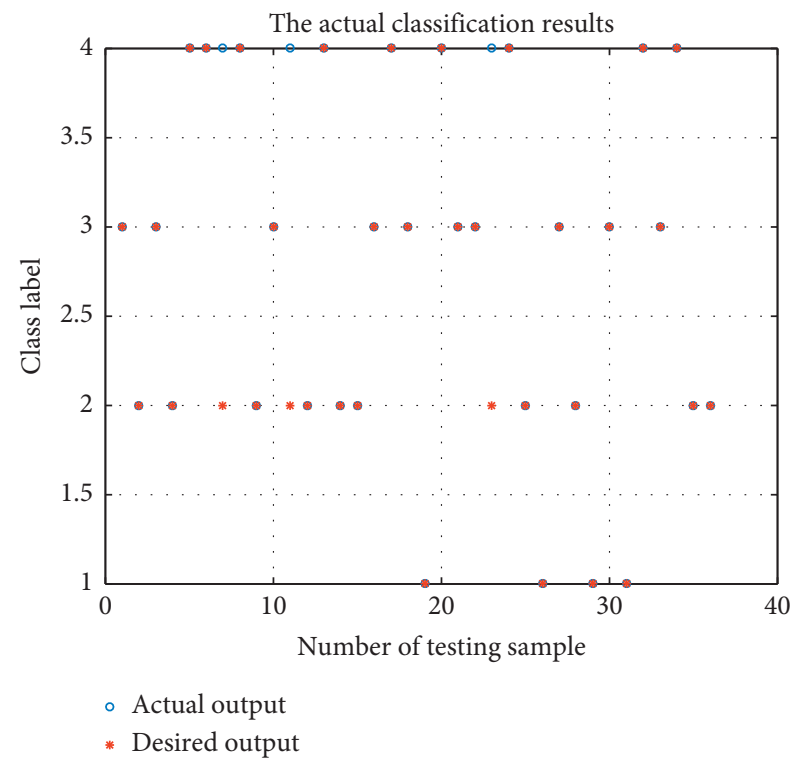

(a)

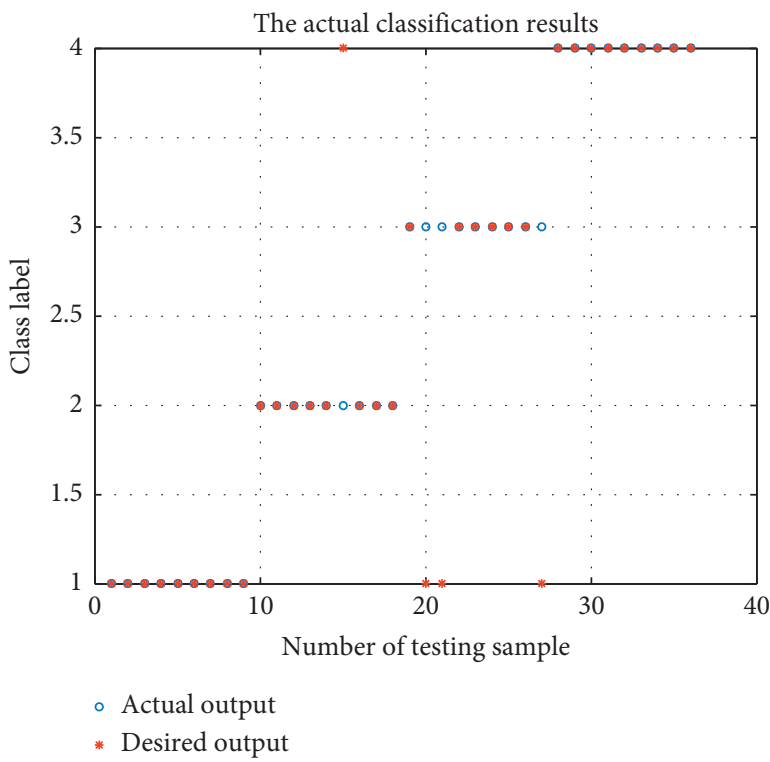

(b)

FIGURE 8: Identification of the numerical simulation based on the power spectrum entropy. (a) SVM model. (b) PSO-SVM model.

rolling element failure, respectively, totaling 160 sets of data, and each set of data samples 2560 points. Among them, $70 \%$ are randomly selected as training samples under different conditions, and the rest $30 \%$ are used as test samples. As the data acquisition is finished, the signal is decomposed by ICEEMDAN to obtain the IMF component series (the example of the outer circle fault is shown in Figure 12). Then, the correlation coefficient-covariance contribution of each order of the IMF components with the original vibration signal is calculated subsequently. It could be observed from Figure 13 that the correlation coefficient-variance contribution rate of the first five-order IMF involves a positive 


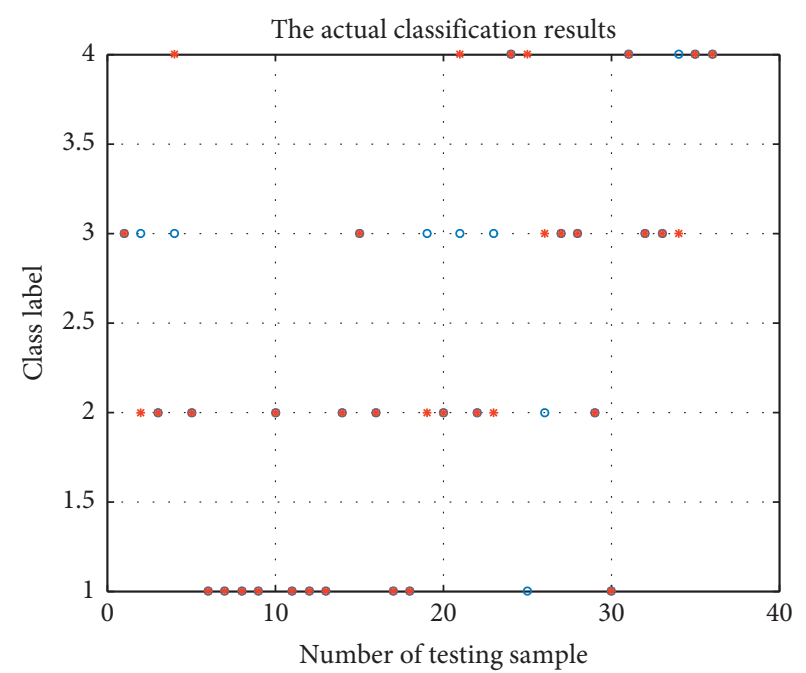

- Actual output

* Desired output

(a)

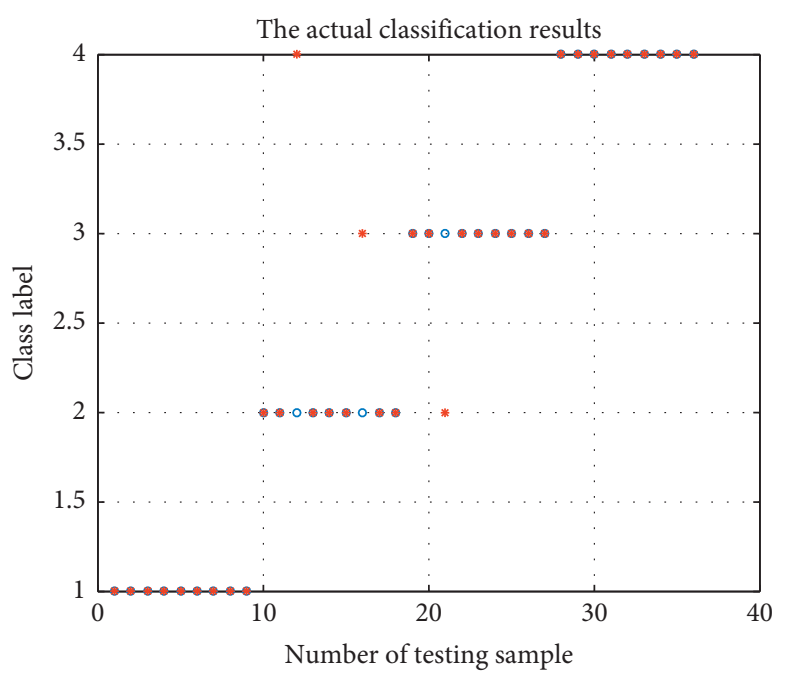

- Actual output

* Desired output

(b)

FIGURE 9: Identification of the numerical simulation based on singular spectral entropy. (a) SVM model. (b) PSO-SVM model.

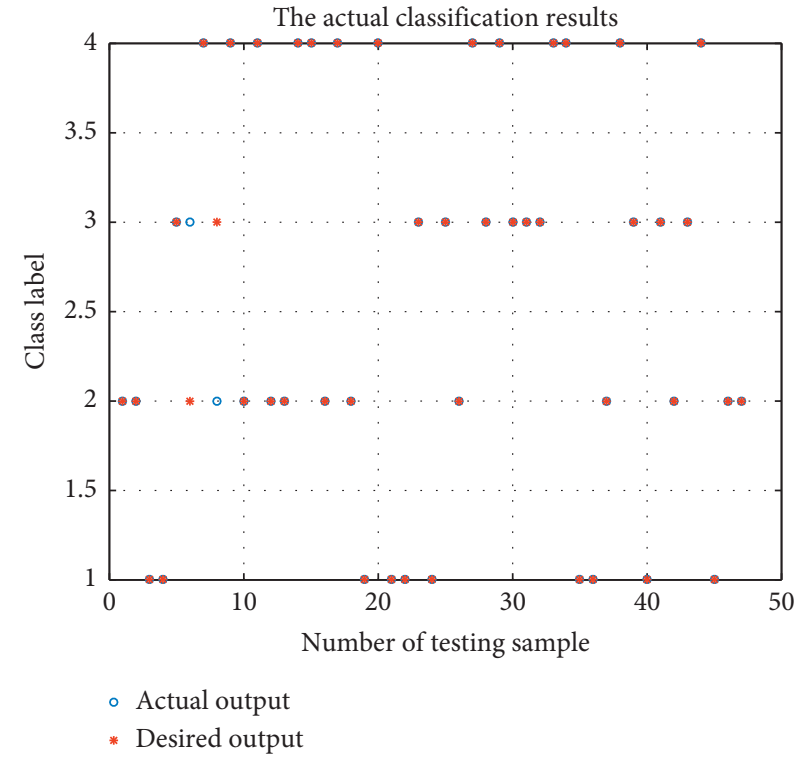

(a)

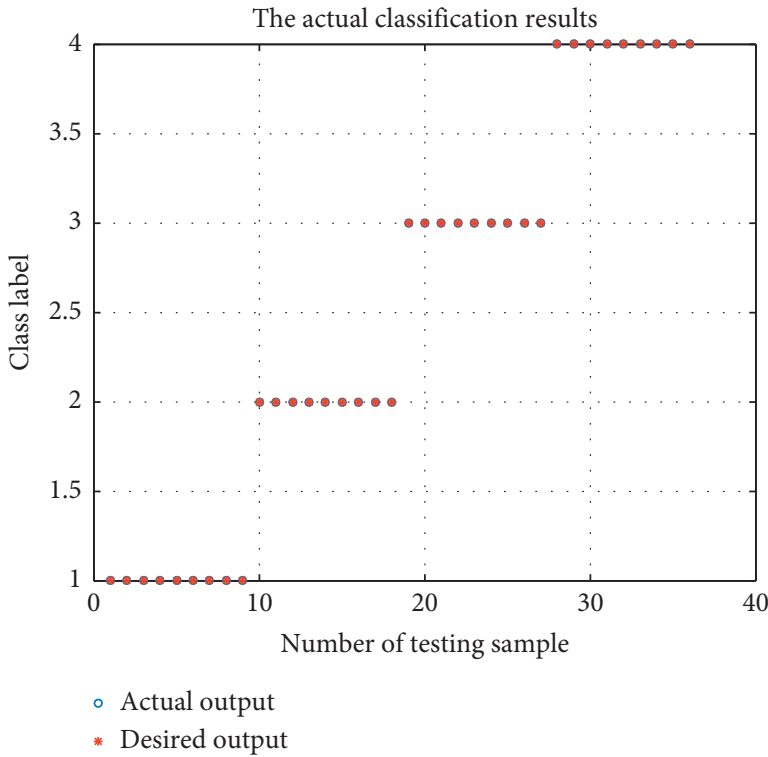

(b)

FIGURE 10: Identification of the numerical simulation based on time-domain energy entropy. (a) SVM model. (b) PSO-SVM model.

trend. The power spectral entropy, singular spectral entropy, and time-domain energy entropy of the first five orders of IMF are also extracted as a set of feature vectors, which are input into the SVM-based model and PSO-SVM-based model, respectively, for pattern recognition. With the analysis processed, the power spectral entropy was selected as a feature as shown in Figure 14. The result shows that the recognition accuracy of the SVM model is $87.5 \%$, while the PSO-SVM model is $93.8 \%$ for the 48 sets of data tested. When singular spectral entropy is selected as a feature, as shown in Figure 15, there are 5 sets of data that are not recognized based on the SVM model, and the accuracy rate is merely $89.6 \%$, and the recognition accuracy rate of the PSO-SVM model reaches 91.7\%; and when the time-domain energy entropy is selected as the feature as shown in Figure 16, the accuracy is $95.8 \%$ based on the SVM model, and the recognition accuracy reaches $100 \%$ based on the PSO-SVM model as well. The results of selecting different feature values and using different classifiers are shown in Table 3. 


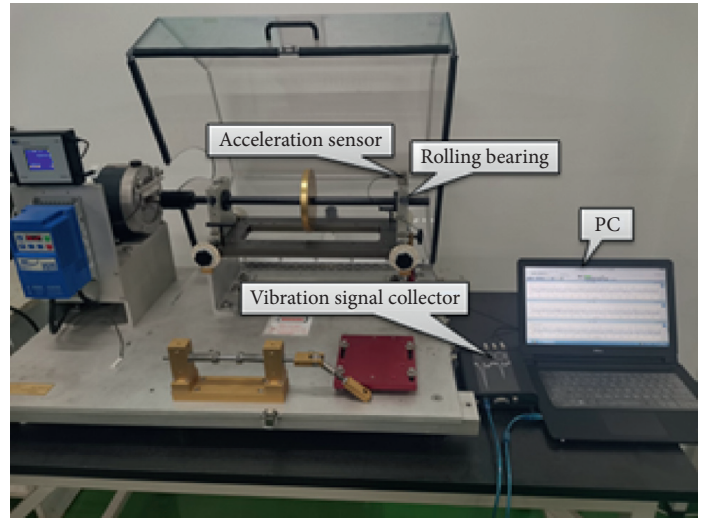

FIgURE 11: Comprehensive mechanical failure simulation test bench.
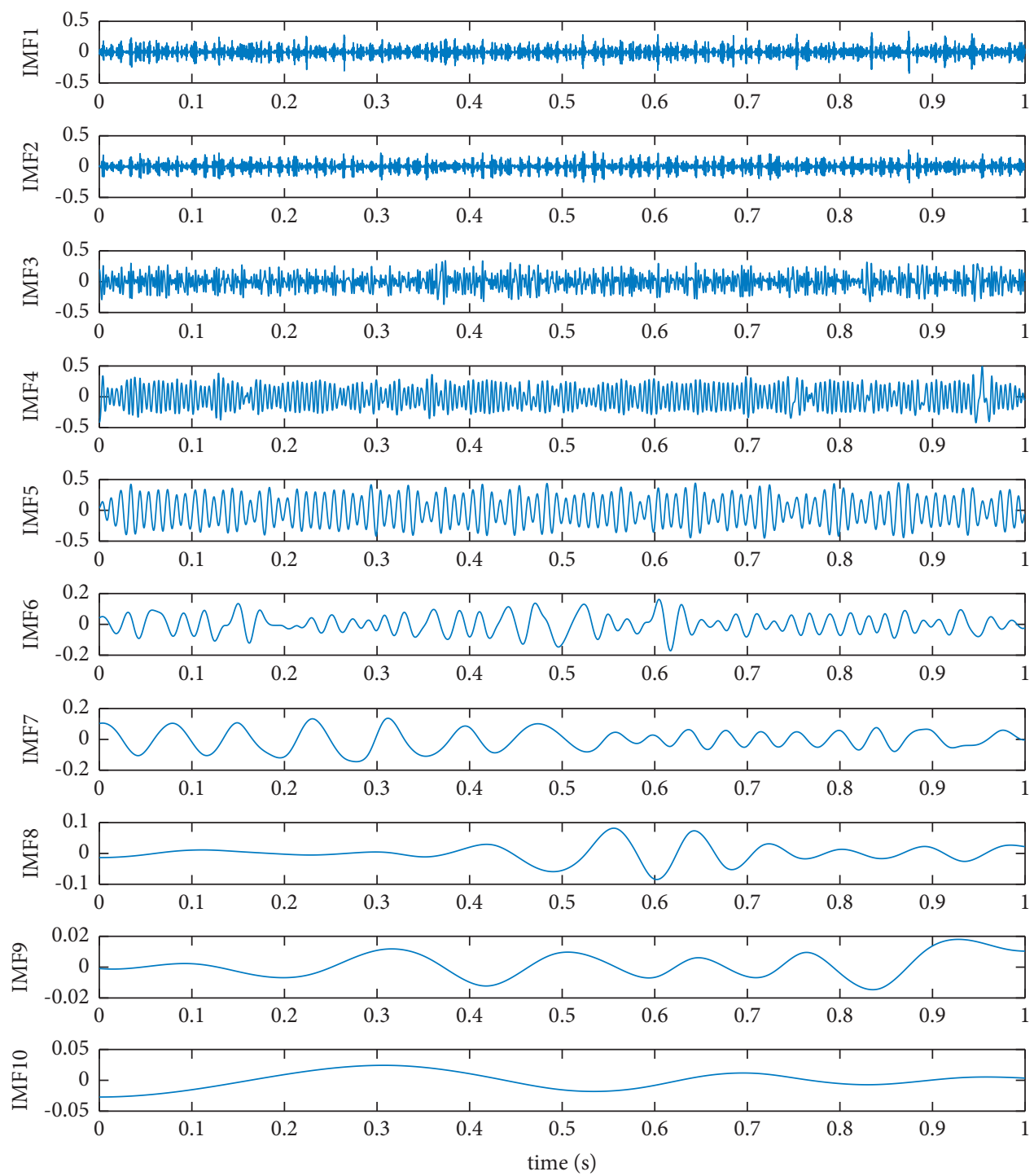

FIGURE 12: ICEEMDAN decomposition of the experimental outer ring fault mode. 


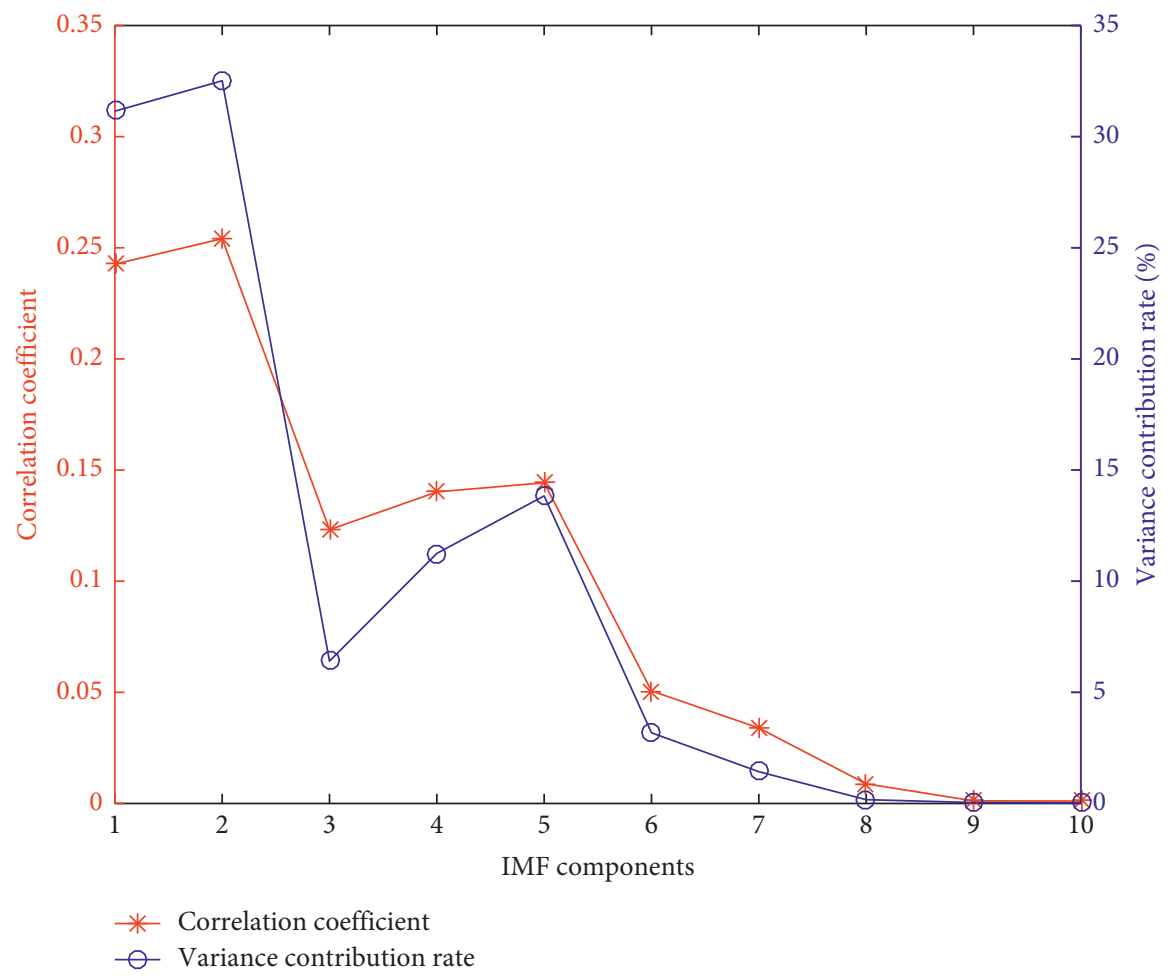

FIGURE 13: Correlation coefficient-variance contribution trend of the experimental outer ring fault mode.

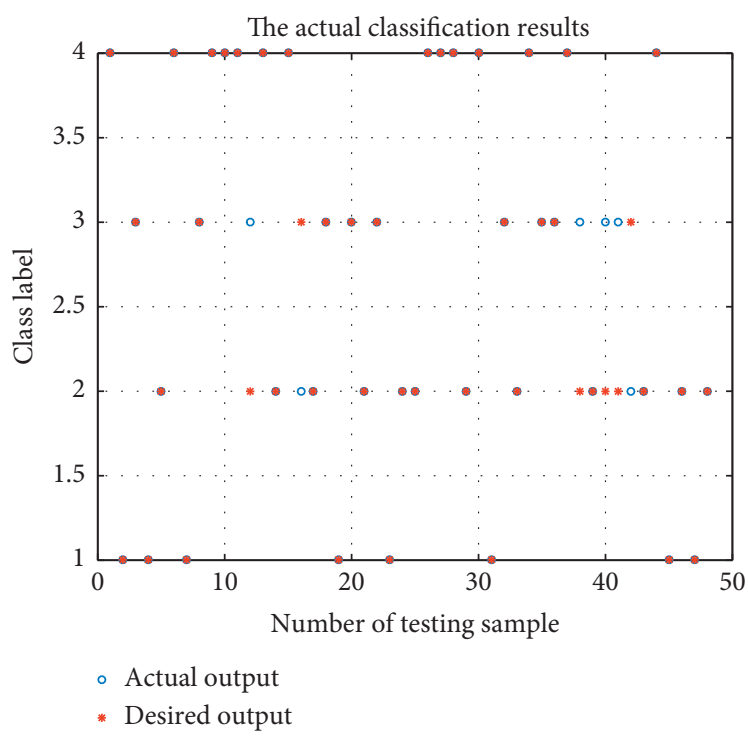

(a)

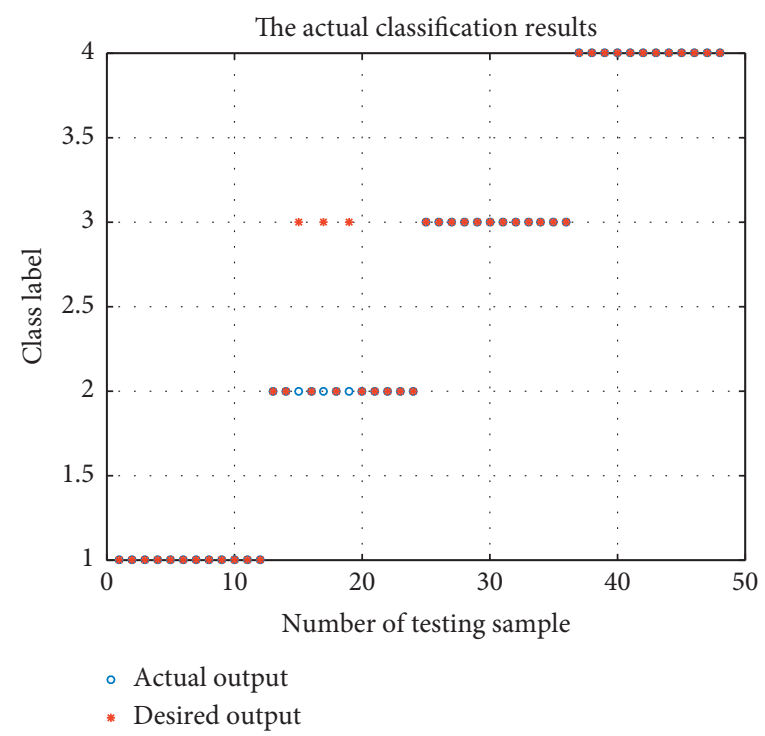

(b)

FIGURE 14: Recognition result of the experimental signal based on power spectrum entropy. (a) SVM model. (b) PSO-SVM model. 


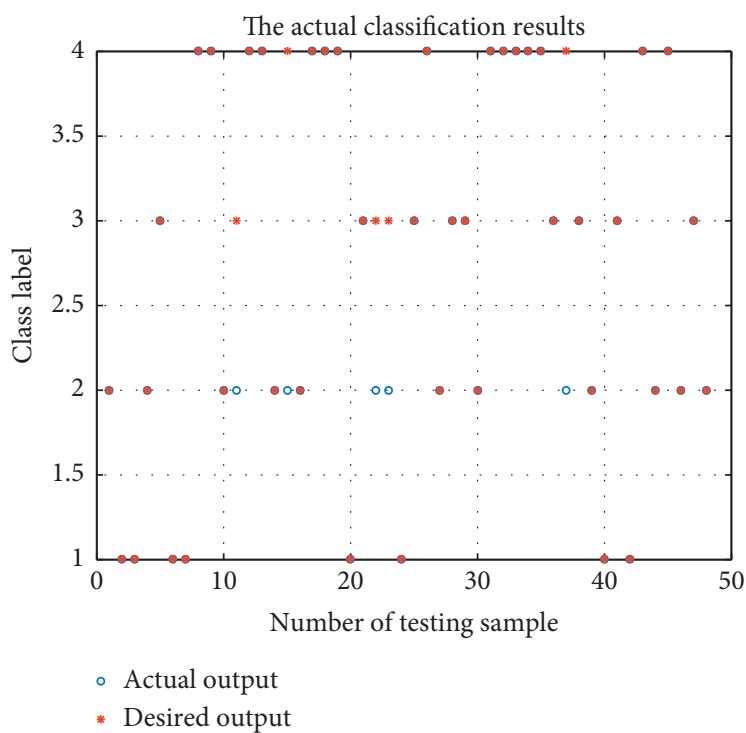

(a)

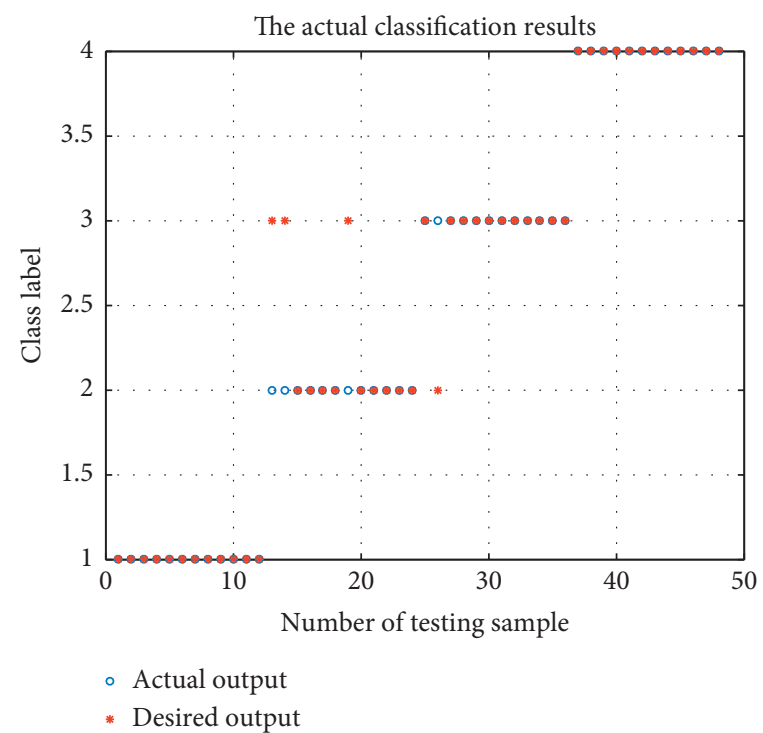

(b)

FIGURE 15: Recognition result of the experimental signal based on singular spectral entropy. (a) SVM model. (b) PSO-SVM model.

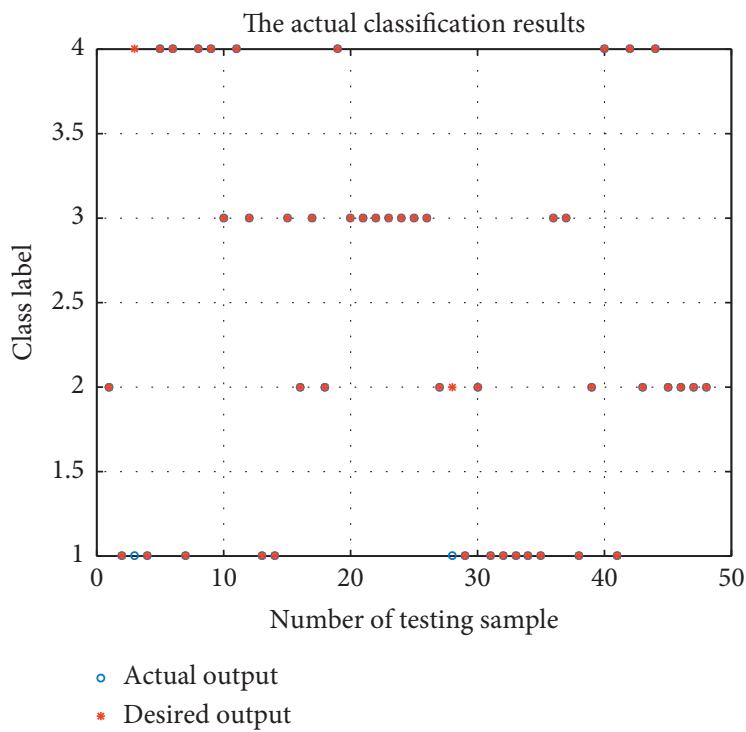

(a)

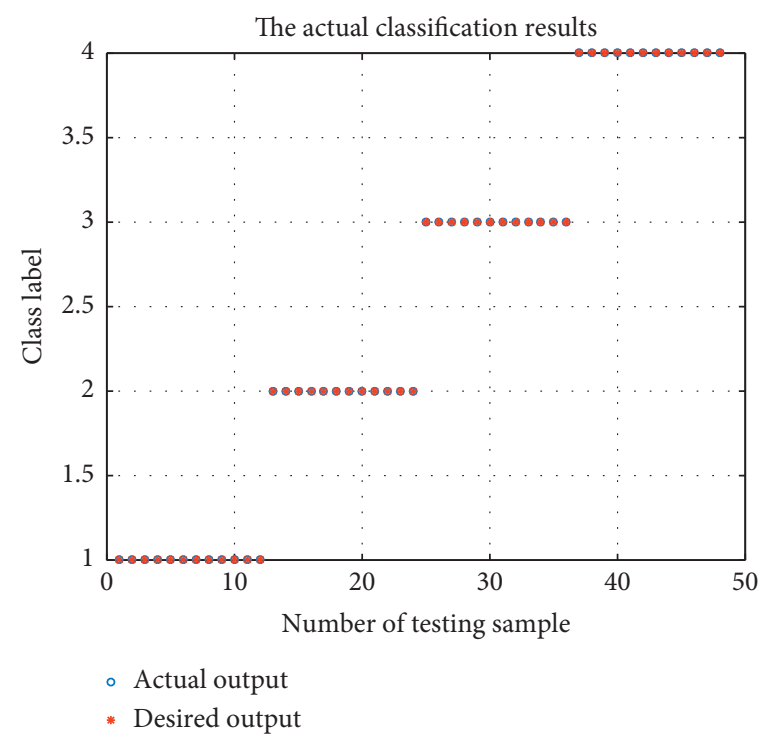

(b)

FIGURE 16: Recognition result of the experimental signal based on time-domain energy entropy. (a) SVM model. (b) PSO-SVM model.

TABLe 3: Results' comparison chart.

\begin{tabular}{|c|c|c|c|c|}
\hline \multirow{2}{*}{ Category } & \multicolumn{2}{|c|}{ Numerical simulation results } & \multicolumn{2}{|c|}{ Experimental results } \\
\hline & SVM $(\%)$ & PSO-SVM (\%) & $\operatorname{SVM}(\%)$ & PSO-SVM (\%) \\
\hline Power spectrum entropy & 91.6 & 88.9 & 87.5 & 93.8 \\
\hline Singular spectral entropy & 77.8 & 91.6 & 89.6 & 91.7 \\
\hline Time-domain energy entropy & 94.4 & 100 & 95.8 & 100 \\
\hline
\end{tabular}


Through experiments, it can be further verified that the fault diagnosis method based on ICEEMDAN decomposition and extracting time-domain energy entropy as a feature, combined with PSO-SVM, is much applicable.

\section{Conclusion}

In this study, a rolling bearing fault pattern recognition method based on the ICEEMDAN time-domain energy entropy model is proposed in order to promote the accuracy of the rolling bearing fault feature extraction method and guarantee the final pattern recognition. The overall study on the validity of the proposed methodology is demonstrated by numerical simulation and experimental analysis for comparison, and the specific conclusions are as follows:

(1) The ICEEMDAN decomposition of the signal is followed by the extraction of useful components using the correlation coefficient-covariance contribution criterion, thus achieving the purpose of dimensionality reduction and optimization of the feature vector set and reducing the computational effort of subsequent pattern recognition.

(2) In terms of feature extraction, three information entropies are employed and compared, i.e., power spectrum entropy, singular spectrum entropy, and time-domain energy entropy. It could be observed that the time-domain energy entropy could embody better with a higher resolution in different states and is more accurate in distinguishing different fault types.

(3) As the feature vector set composed of the three extracted information entropies is input into the SVM model and PSO-SVM model for identification, the accuracy was $94.4 \%$ for the numerical simulation based on the SVM model and $95.8 \%$ for the experiment, while the accuracy rate of the PSO-SVM model could reach $100 \%$, which indicates that the PSO-SVM model performs better in recognition accuracy of the rolling bearing fault mode.

\section{Data Availability}

The data used to support the findings of this study are available from the corresponding author upon request.

\section{Conflicts of Interest}

The authors declare that they have no conflicts of interest.

\section{Acknowledgments}

This research was supported by the Project of the National Natural Science Foundation of China (no. 52075050), Natural Science Research Project of Universities in Jiangsu Province (no. 19KJA430004), and Jiangsu Key Laboratory of Green Process Equipment.

\section{References}

[1] X. Zhao and M. Jia, "Fault diagnosis of rolling bearing based on feature reduction with global-local margin Fisher analysis," Neurocomputing, vol. 315, pp. 447-464, 2018.

[2] N. Rodriguez, P. Alvarez, L. Barba, and G. Cabrera, "Combining multi scale wavelet entropy and kernelized classification for bearing multi-fault diagnosis," Entropy, vol. 21, no. 2, pp. 15-25, 2019.

[3] C. Peeters, P. Guillaume, and J. Helsen, "A comparison of cepstral editing methods as signal pre-processing techniques for vibration-based bearing fault detection," Mechanical Systems and Signal Processing, vol. 91, no. 1, pp. 354-381, 2017.

[4] X. Yu, F. Dong, E. Ding, S. Wu, and C. Fan, "Rolling bearing fault diagnosis using modified LFDA and EMD with sensitive feature selection," IEEE Access, vol. 6, no. 99, pp. 3175-3730, 2018.

[5] Y. X. Wang, Z. J. He, and Y. Y. Zi, "A comparative study on the local mean decomposition and empirical mode decomposition and their applications to rotating machinery health diagnosis," Journal of Vibration and Acoustics, vol. 132, no. 2, 2010.

[6] C. Wahyu, B. K. Buyung, and K. T. Anh, "Condition monitoring of naturally damaged slow speed slewing bearing based on ensemble empirical mode decomposition," Journal of Mechanical Science and Technology, vol. 27, no. 8, pp. 22532262, 2013.

[7] Z. Wu and N. E. Huang, "Ensemble empirical mode decomposition: a noise-assisted data analysis method," Advances in Adaptive Data Analysis, vol. 1, no. 1, 2009.

[8] M. E. Torres, M. A. Colominas, and G. Schlotthauer, "A complete ensemble empirical mode decomposition with adaptive noise," in Proceedings of the IEEE International Conference on Acoustics Speech and Signal Processing, pp. 4144-4147, IEEE, Prague, Czech Republic, May 2011.

[9] Y. Lei, Z. Liu, J. Ouazri, and J. Lin, "A fault diagnosis method of rolling element bearings based on CEEMDAN," Proceedings - Institution of Mechanical Engineers, vol. 231, no. 10, pp. 1989-1996, 2017.

[10] B. Keshtegar, M. Bagheri, C. W. Fei, L. Chen, T. Osman, and T. Dui-Kien, "Multi-extremum-modified response basis model for nonlinear response prediction of dynamic turbine blisk," Engineering with Computers, Article ID 101007, 2021.

[11] X. Zhang, J. M. Zhao, H. P. Li, X. L. Ni, and F. C. Sun, "Gearbox hybrid fault diagnosis based on NIC-DWT-WOASVM," Vibration and shock, vol. 39, no. 11, pp. 146-151, 2020.

[12] B. W. Wen, W. H. Dong, and W. J. Xie, "Random forest parameter optimization based on improved grid search algorithm," Computer Engineering and Applications, vol. 54, no. 10, pp. 154-157, 2018.

[13] H. J. Liu, C. Chen, Z. Q. Guo, Y. Xia, X. Yu, and S. Li, “Overall grouting compactness detection of bridge prestressed bellows based on RF feature selection and the GA-SVM model," Construction and Building Materials, vol. 301, Article ID 124323, 2021.

[14] W. B. Yang, B. P. Gao, F. Chen, X. Zhang, and W. Ma, "Fault diagnosis of crane gearbox based on variational modal decomposition and PSO-SVM," Mechanical Transmission, vol. 45, no. 4, pp. 105-111, 2021.

[15] Q. Fang, Q. Y. Liu, and T. Liu, "Rolling bearing fault diagnosis method based on hybrid domain feature set and PSO-SVM," Mechanical Engineering and Automation, vol. 2, pp. 1-3, 2020.

[16] M. Zhu, Z. S. Duan, and B. L. Guo, "Analysis of noise reduction effect of vibration screen bearing signal based on 
EEMD combined with wavelet packet," Machinery Design and Manufacturing, vol. 3, pp. 63-67, 2020.

[17] G. J. Tang, S. C. Li, S. Y. Lu, W. J. Zhang, and X. Gao, "Rotor fault diagnosis based on CERLMDAN," Chinese Journal of Power Engineering, vol. 40, no. 9, pp. 720-727, 2020.

[18] M. A. Colominas, G. Schlotthauer, and M. E. Torres, "Improved complete ensemble EMD: a suitable tool for biomedical signal processing," Biomedical Signal Processing and Control, vol. 14, no. 1, pp. 19-29, 2014.

[19] L. L. Jiang, H. C. Tan, and X. J. Li, "Spiral bevel gear fault identification based on CEEMDAN permutation entropy and SVM," Vibration, Testing and Diagnosis, vol. 41, no. 1, pp. 33-40+198, 2021.

[20] H. Yin, Q. Chen, and Z. R. Peng, "Gearbox bearing fault feature extraction based on optimal sensor placement," Noise and Vibration Control, vol. 40, no. 4, pp. 67-72+154, 2020.

[21] C. W. Fei, G. C. Bai, W. Z. Tang, and M. Shuang, "Quantitative diagnosis of rotor vibration fault using process power spectrum entropy and support vector machine method," Shock and Vibration, vol. 2014, Article ID 957531, 9 pages, 2014.

[22] X. Zhang, C. Mei, D. Chen, and J. Li, "Feature selection in mixed data: a method using a novel fuzzy rough set-based information entropy," Pattern Recognition, vol. 56, no. 1, pp. 1-15, 2016.

[23] J. M. Zhou, F. L. Wang, and C. C. Zhang, "Intelligent evaluation method for rolling bearings based on feature optimization and GA-SVM," Vibration and Shock, vol. 40, no. 4, pp. 227-234, 2021.

[24] B. Li and Y. Min, "Analysis model of drilling tool failure based on PSO-SVM and its application," in Proceedings of the Fourth International Conference on Computational \& Information Sciences IEEE, pp. 1307-1310, Chongqing, China, August 2012.

[25] J. C. Liu, H. T. Wang, S. P. Zeng, and Z. G. Yuan, "Intelligent evaluation model for cementing quality based on PSO-SVM and application," Applied Mechanics and Materials, vol. 71-78, no. 2, pp. 4293-4299, 2011.

[26] J. P. Zhang, Y. J. Li, and Y. G. Li, "ADAMS simulation and experimental research on the wear state of rolling bearings," Mechanical Design and Manufacturing, vol. 6, pp. 112-115, 2019.

[27] G. Zhang, Y. H. He, and Y. M. Zhang, "Multi-body contact dynamics simulation of rolling bearings," Vibration. Testing and Diagnosis, vol. 33, no. S1, pp. 28-31, 2013. 